Vol.45, n. 3 : pp. 343-353, September 2002

ISSN 1516-8913 Printed in Brazil

\title{
Check-list of the Family Lamiaceae in Pernambuco, Brazil
}

\author{
Cecília de Fátima C. B. R. de Almeida and Ulysses Paulino de Albuquerque* \\ Laboratório de Etnobotânica e Botânica Aplicada (LEBA); Departamento de Botânica; Centro de Ciências \\ Biológicas; Universidade Federal de Pernambuco; upa@npd.ufpe.br; Recife - PE - Brazil
}

\begin{abstract}
A survey of the family Lamiaceae in the state of Pernambuco revealed the presence of 20 genera encompassing 46 species. The genera with the highest number of exsiccatae were Hyptis and Ocimum; others had little representative material, and some taxa were collected only once. Regarding the phytogeographical distribution in the state, 9.1\% of the registrations of species occurred in the coastal zone, $54.5 \%$ in the forest zone and $36.3 \%$ in the caatinga zone.
\end{abstract}

Key words: Lamiaceae, floristic survey, check-list

\section{INTRODUCTION}

The family Lamiaceae includes approximately 220 genera (Hedge, 1992) and 3.500 to 4.000 species (Harley, 1988; Hedge, 1992). With wide distribution, it has cosmopolitan representatives (Salvia and Scutellaria), and is especially abundant in the Mediterranean region. It grows in several habitats and altitudes: from the Arctic regions to the Himalayas, southeast Asia to Hawaii, Australia, Africa, and the New World from north to south (Heywood, 1978). It is a typical savanna family, inhabiting warm areas around the world (Harley, 1988).

In northeastern Brazil, floristic and taxonomic information on the family Lamiaceae is very sparse, consisting mainly of records of new species. With exception of the contributions of Harley $(1995,1996)$ for the state of Bahia, little is known about the species occurring in the states of the northeast region. Specifically in Pernambuco, the family has not yet received a systematic treatment, with records sparsely distributed in a vast literature of mainly floristic surveys. Among others, the following species are reported for the state of Pernambuco: Hyptis fruticosa Salzm. (Andrade-Lima, 1960), H. lantanifolia Poit. (Andrade-Lima, 1966), H. suaveolens (L.) Poit., Leonotis nepetifolia R. Br. (Silva, 1985), Scutellaria agrestis St. Hil. (Epling and Toledo, 1943), Hyptis lappulacea Mart. ex Benth., $H$. pectinata (L.) Poit., Leucas martinicensis R.Br (Pereira, 1965), Marsypianthes chamaedrys (Vahl) Kuntze (Pereira and Pereira, 1973), and the genus Ocimum, which is receiving a series of treatments (Albuquerque and Andrade, 1998a,b; Albuquerque, 1998, 1999).

In this work a preliminary survey on the family Lamiaceae for Pernambuco state is presented. Data on geographic distribution of the recorded species is also provided.

\footnotetext{
${ }^{*}$ Author for correspondence
} 


\section{MATERIALS AND METHODS}

The present research was based on the analysis of material collected in the state of Pernambuco, as only the material identified and/or confirmed by specialists of the family has been included. The examined exsiccatae were obtained from herbaria and listed with the respective abbreviation according to Index Herbariorum (Holmgreen et al., 1990): IPA (Herbarium "Professor Dárdano de Andrade Lima), of the Empresa Pernambucana de Pesquisa Agropecuária, Recife, Pernambuco, Brazil; PEUFR (Herbarium "Professor Vasconcelos Sobrinho"), Departamento de Biologia of the Universidade Federal Rural de Pernambuco, Recife, Brazil; UFP (Herbarium "Professor Geraldo Mariz"), Departamento de Botânica, Centro de Ciências Biológicas of the Universidade Federal de Pernambuco, Recife, Brazil.

The data on physical environment, habitat, plant habit, vernacular names and other information, when available, were obtained from exsiccatae labels. The material examined was cited according to subfamily. Each genus was listed by species, with locality, collection date, name and number of collector and abbreviation of herbaria. The accession number of the herbarium identifies the specimens with no collector number. Cultivated or exotic species are marked with an asterisk.

A brief analysis of the geographical distribution of the species in the state of Pernambuco was carried out, excluding the cultivated species. The phytogeographic division of Pernambuco as proposed by Andrade-Lima (1960), was adopted. The data on general distribution in Brazil and in the world, were obtained according to available literature.

\section{RESULTS AND DISCUSSION}

In Pernambuco the family has been represented by 46 species within 20 genera: Aeollanthus (1 sp), Eriope (2 spp), Hesperozygis (1 sp), Hypenia (1 $\mathrm{sp})$, Hyptis (17 spp), Leonotis (1 sp), Leonurus (1 $\mathrm{sp})$, Leucas (1 sp), Marsypianthes (1 sp), Mentha (2 spp), Ocimum (7 spp), Plectranthus (2 spp), Pogostemon (1 sp), Raphiodon (1 sp), Rosmarinus (1 sp), Salvia (2 spp), Satureja Scutellaria (1 sp), Solenostemon (1 sp) and Tetradenia (1 sp). About 39\% of these are exotic and/or cultivated species. The species Leonotis nepetifolia (L.) R. Br., Leonurus japonicus Houtt and Leucas martinicensis L., are cosmopolitan, occurring as ruderals and as weeds in Brazilian territory.

The genus Hesperozygis was found in the southern states of Brazil and has been never reported for the Northeast. It was the result of only one collection at the Campus of the Universidade Federal Rural de Pernambuco in 1979, and was identified by the specialist R.M. Harley, who stressed the particularity of its distribution. However, there are still too few collections for Pernambuco, with a number of taxa that were collected only once, such as Satureja sp., Scutellaria agrestis St. Hil. and Leonurus japonicus Houtt.

The tribe Ocimeae of the subfamily Nepetoideae was the best represented. The genera Ocimum and Hyptis were the most representative with respect to the number of species. Hyptidinae was represented by five genera: Hyptis, Hypenia, Eriope, Marsypianthes and Raphiodon (monotypical genus of eastern Brazil). In the study carried out by Harley (1996) the two largest genera in Bahia were Hyptis (51) and Eriope (17). Harley (1996) mentioned that the genus Eriope is typically Brazilian, particularly on rupestrian fields, having its center of diversity in the Chapada Diamantina, with secondary centers in Minas Gerais and Goiás. In Pernambuco this genus was represented by two wide distributed species: Eriope crassipes Benth. and $E$. cf. macrostachya Mart. ex Benth.

With respect to geographical distribution, $9.1 \%$ of species occurred in the coastal zone, $54.5 \%$ in the forest zone and $36.3 \%$ in the caatinga zone. There was a higher number of collections for the forest zone. The genera with the widest distribution in Pernambuco were: Hypenia, Hyptis, Marsypianthes, Leonotis, Ocimum and Raphiodon. The genus Hyptis occurs from the coastal zone to the caatinga. The collections for the coastal zone also include many specimens of the genus Ocimum.

The species and examined material are listed below:

\section{Subfamily Scutellarioideae Scutellaria L. \\ Scutellaria agrestis St. Hil.}

It occurs in north and central Brazil, from north Minas Gerais to Pernambuco (Epling and Toledo, 1943). Occurs in the forest zone of Pernambuco. 
Material examined: PERNAMBUCO: Tapera, Ipojuca, VIII/1930, Pickel s.n., IPA 4797.

\section{Subfamily Lamioideae}

Leonotis (Pers.) R. Br.

Leonotis nepetifolia (L.) R. Br. *

Vernacular: Cordão-de-frade, cordão-de-SãoFrancisco.

It inhabits open and dry areas throughout Brazil. In Pernambuco occurs from the coastal zone to the caatinga zone.

Material examined: PERNAMBUCO: Buíque, Vegetação arbustiva densa, 18/VIII/1995, Andrade 160 and Figueredo, PEUFR 22699. Carpina, Campus do CENTREINO-EMATER, 23/VIII/1986, Lima et al. 11, IPA 48964. Idem, 1986, Bedi 4, IPA 51412. Caruaru, 25/IV/1995, Albuquerque 45, UFP 11035. Idem, 25/IV/1995, Albuquerque 44, UFP 11036. Floresta, Estrada Ibimirim-Floresta, XI/1985, Ataide et al. 684, IPA 48946. Idem, XI/1985, Ataide et al s.n., PEUFR 8523. Gravatá, Serra das Russas, 30/VIII/1959, Tavares 515, UFP 325. Ipojuca, Área Projeto Suape, 23/XI/1977, Andrade-Lima and Medeiros-Costa 96, IPA 22292. Maraial, 24/IV/1997, Baracho 555 and Siqueira 545, UFP 18671. Olinda, VII/1929, Pickel s.n., IPA 4798. Recife, 26/IX/1997, Conceicão 9, PEUFR 22575. Idem, Cidade Universitária, 3/V/1984, Silva s.n., UFP 4280. São Lourenço da Mata Engenho Bela Rosa, 1/IV/1982, Chiappeta 118, IPA 32018. Idem, 13/IX/1983, Chiappeta et al. s.n., IPA 32142. Idem, 13/IX/1983, Chiappeta et al. s.n., PEUFR 7269. Taquaritinga do Norte, 7/VI/1995, Albuquerque 76, UFP 11050.

\section{Leonurus $\mathrm{L}$.}

\section{Leonurus japonicus Houtt}

= Leonurus sibiricus auct. non $\mathbf{L}$. *

Vernacular: Cordão-de-frade.

It is native to Asia (Corrêa, 1969). Occurs in forest zone of Pernambuco, and much of northeast Brazil.

Material examined: PERNAMBUCO: Tapera, Ipojuca, 23/VIII/1934, Pickel s.n., IPA 7011.

\section{Leucas R. Br.}

Leucas martinicensis $\mathrm{L}$. *

Vernacular: Cordão-de-frade.

Native to Equatorial Africa, and is perhaps the Lamiaceae with the largest area of dispersion (Epling and Toledo, 1943). Widely distributed throughout Brazil. Occurs in forest zone of Pernambuco.

Material examined: PERNAMBUCO: Carpina, 1986, Bedi 5, IPA 51413. Tapera, Ipojuca, VII/1930, Pickel s.n., IPA 4800.

\section{Subfamily Nepetoideae}

Tribe Mentheae

\section{Mentha $\mathbf{L}$.}

Mentha arvensis $\mathbf{L}$. *

Vernacular: Hortelã.

It is an introduced plant from Europe, well adapted to South America. Cultivated for medicinal purposes.

Material examined: PERNAMBUCO: Olinda, Centro Nordestino de Medicina Popular, VII/1998, Santana s.n., IPA 57048.

\section{Mentha pulegium L. *}

Vernacular: Poejo.

Plant of European origin, well adapted to South America (Lainetti and Brito, 1980). It may also be found in Occidental Asia and Septentrional Africa (Corrêa, 1974; Morgan, 1997). Cultivated as medicinal in throughout Pernambuco.

Material examined: PERNAMBUCO: Recife, Ibura, 19/VIII/1991, Krusch s.n., PEUFR 11537.

\section{Salvia L.}

Salvia farinacea Benth. *

Vernacular: Salvia.

It is native to Mexico (Corrêa, 1975). Cultivated as ornamental in Pernambuco.

Material examined: PERNAMBUCO: Correntes, XI/1929, Pickel s.n., IPA 4802. Recife, 28/IX/1965, Vasconcelos Sobrinho 10, PEUFR 9875.

\section{Salvia splendens Sellow}

Vernacular: Cardeal-do-Brasil, sangue-de-Adão, pé-de-chumbo.

Ornamental species, exclusively from Brazil. Much cultivated by horticulturists of Europe. In Pernambuco it is widely used in gardening.

Material examined: PERNAMBUCO: Recife, Graças, s.d., Leão s.n., UFP 1511. Idem, Dois Irmãos, Silva 
s.n., UFP 1544. Tapera, Campo Alegre, Ipojuca, 18/IX/1931, Pickel s.n., IPA 4803.

\section{Hesperozygis Epling Hesperozygis sp.}

It is a genus widely distributed in southern Brazil, without references for the Northeast. According to Pereira and Pereira (1973) five species occur in Brazil. It occurs in forest zone of Pernambuco.

Material examined: PERNAMBUCO: Recife, Dois Irmãos, 21/X/1979, Barreto 11, PEUFR 5939.

\section{Rosmarinus L.}

Rosmarinus officinalis L. *

Vernacular: Alecrim, alecrim-de-cheiro.

Native to South Europe, it also occurs in North Africa and in the Middle East (Castro and Chemale, 1995).

Material examined: PERNAMBUCO: Limoeiro, Lagoa Funda, 21/XI/1928, Pickel s.n., IPA 4796. Recife, 27/II/1985, Pires 7, UFP 4275. Tapera, Ipojuca, XII/1926, Pickel s.n., IPA 7007.

\section{Satureja sp.}

\section{Satureja $\mathbf{L}$.}

It was found in forest zone of Pernambuco.

Material examined: PERNAMBUCO: Tapera, Ipojuca, 9/XI/1932, Pickel s.n., IPA 7013.

\section{Tribe Ocimeae}

Ocimum L.

Ocimum americanum L. *

Vernacular: Manjericão, manjerona.

Plant of African origin, widespread in Tropical America and cultivated throughout Brazil (Corrêa, 1974; Albuquerque and Andrade, 1998a).

Material examined: PERNAMBUCO: Afrânio, 21/IV/1971, Heringer et al. s.n., IPA 19105. Idem, 21/IV/1971, Heringer et al. s.n., PEUFR 4229. Carpina, 22/IV/1997, Lima and Rita s.n., IPA 50866. Macaparana, 7/VI/1995, Albuquerque 84, UFP 13882. Olinda, 1915, Pickel s.n., IPA 4830. Recife, Margem do Capibaribe, III/1938, Vasconcelos Sobrinho s.n., IPA 672. Idem, Cidade Universitária, 24/V/1985, Silva s.n., UFP 4403. Idem, Cidade Universitária, s.d., Costa s.n., UFP 1638. Russinha, Pombos, 6/VI/1934, Pickel s.n., IPA 4832. Serra Talhada, 10/V/1971, Heringer et al. s.n., IPA 19540. Tapera, Ipojuca, 4/X/1932, Pickel s.n., IPA 4831. Idem, IX/1929, Pickel s.n., IPA 4812. Vitória de Santo Antão, BR-25, Km 48, 11/VII/1961, Tavares 651, UFP 515.

\section{Ocimum basilicum L. *}

Vernacular: Alfavaca, alfavaca-cheirosa, manjericão, manjericão-graúdo.

Of African origin, this plant is cultivated throughout Brazil (Albuquerque and Andrade, 1998a).

Material examined: PERNAMBUCO: Bodocó, 2/V/1978, Fernandes and Matos s.n., PEUFR 11295. Carpina, 22/IV/1987, Lima and Rita s.n., IPA 50872. Idem, 22/IV/1987, Lima and Rita s.n., IPA 50873. Caruaru, Murici, 25/IV/1995, Albuquerque 48, UFP 11291. Idem, Murici, 25/IV/1995, Albuquerque 49, UFP 11291. Goiana, 13/II/1990, Lima 525, IPA 52497. Idem, 13/II/1990, Lima 527, IPA 52499. Olinda, VIII/1998, Santana s.n., IPA 57065. Pesqueira, Jardim Residencial, 26/IV/1995, Albuquerque 56, UFP 11293. Poção, PE 197, 26/IV/1995, Albuquerque 54, UFP 11292. Idem, PE 197, 26/IV/1995, Albuquerque 55, UFP 13721. Recife, 12/V/1993, Silva s.n., PEUFR 14328. Idem, Cidade Universitária, I/1985, Pires 1, UFP 4264. Idem, Arruda, 21/IV/1993, Albuquerque s.n., UFP 8754. Idem, Dois Irmãos, 1/VI/1993, Albuquerque s.n., UFP 8762. Idem, 14/X/1994, Barros and Silva s.n., UFP 10720. Tapera, Ipojuca, IX/1929, Pickel s.n., IPA 4812.

\section{Ocimum campechianum Mill.}

Vernacular: Alfavaca, alfavaca-brava, manjericão, manjericão-branco.

Plant widespread through Tropical America, including Brazil. Cultivated and common in the Caatinga (Albuquerque and Andrade, 1998a).

Material examined: PERNAMBUCO: Afrânio, 21/IV/1971, Heringer et al. s.n., IPA 19103. Idem, 11/VI/1993, Heringer et al. s.n., PEUFR 4236. Alagoinha, Fazenda Cajueiro, 27/IV/1995, Albuquerque 69, UFP 11286. Idem, Fazenda Cajueiro, s.d., Albuquerque 35, UFP 10438. Carpina, 1986, Bedi 132, IPA 51600. Caruaru, Murici, Jardim Residencial, 25/IV/1995, Albuquerque 52, UFP 11288. Idem, Murici, 25/IV/1995, Albuquerque 53, UFP 11289. Idem, Murici, 25/IV/1995, Albuquerque 51, UFP 11290. Goiana, 14/VII/1992, Azevedo 18, IPA 53314. Parnamirim, 18/VI/1984, Araújo 160, IPA 42699. Idem, 18/VI/1984, Araújo 160, PEUFR 7471. Idem, Km 27, 18/VI/1984, Araújo 160, UFP 6339. Paulista, Pedreira de São Bento, 14/IX/1961, Tavares 800, UFP 
678. Idem, Arthur Lundgren II, 2/V/1995, Albuquerque 71, UFP 13723. Recife, 1979, Andrade-Lima et al. 799218, IPA 46496. Idem, Dois Irmãos, 1/VI/1990, Bernardo Filho s.n., PEUFR 10349. Idem, Dois Irmãos, 13/V/1991, Krusch s.n., PEUFR 11543. Idem, Dois Irmãos, 3/V/1991, Krusch s.n., PEUFR 11539. Idem, Cidade Universitária, 8/III/1985, Pires 9, UFP 4278. Saltinho, Rio Formoso, 2/IX/1954, Falcão et al. 913, IPA 7876. São Lourenço da Mata Reserva Ecológica de Tapacurá, 6/VI/1995, Albuquerque 72, UFP 11285. Idem, Tapacurá, 6/VI/1995, Albuquerque 73, UFP 11287. Serra Talhada, 17/IV/1971, Heringer et al. s.n., IPA 18862. Idem, 15/IV/1986, Ataide 653, IPA 48777. Tapera, Ipojuca, IX/1927, Pickel s.n., IPA 4835. Idem, VIII/29, Pickel s.n., IPA 4836. Triunfo, 25/II/1986, Lima and Gallindo 11, IPA 48391.

\section{Ocimum gratissimum $\mathbf{L}$. *}

Vernacular: Alfavaca, alfavaca-de-caboclo, louro, louro-cheiroso.

Plant widespread in Tropical America, native of Africa (Albuquerque and Andrade, 1998a). Common as ruderal in Pernambuco.

Material examined: PERNAMBUCO: Caruaru, Vertentes, 30/XI/1927, Pickel s.n., IPA 4833. Idem, Murici, 25/IV/1995, Albuquerque 50, UFP 13722. Idem, Brejo dos Cavalos, 25/IV/1995, Albuquerque 47, UFP 11283. Goiana, 30/V/1935, Pickel s.n., IPA 4834. Gravatá, 9/VII/1965, Guedes 20, PEUFR 9879. Maraial, 28/II/1966, Guedes 57, PEUFR 9876. Idem, 9/VII/1965, Teixeira 2746, PEUFR 9877. Olinda, Centro Nordestino de Medicina Popular, VIII/1998, Santana s.n., IPA 57042. Idem, Bairro Novo, 8/IX/1994, Barbosa 2, UFP 10719. Pombos, 1/I/1929, Pickel s.n., IPA 7005. Recife, Graças, 30/VIII/1989, Lima s.n., PEUFR 10556. Idem, Ibura, 10/V/1991, Krusch s.n., PEUFR 11544. Idem, Torre, Terreno Baldio, 20/X/1976, Correia 9, UFP 4144. Idem, Água Fria, 29/V/1984, Silva s.n., UFP 4217. Idem, Cidade Universitária, II/1985, Pires 5, UFP 4274. Idem, Curado, Jardim Botânico, 21/VIII/1989, Lemos 2, UFP 7738. Idem, Cidade Universitária, 23/VI/1993, Balbino s.n., UFP 8964. Idem, Cidade Universitária, s.d., Santos s.n., UFP 10900. São Lourenço da Mata Reserva Ecológica do Tapacurá, 6/VI/1995, Albuquerque 74, UFP 11284. Taquaritinga do Norte, 7/VI/1995, Albuquerque 77, UFP 11280. Triunfo, Próximo divisa com a Paraíba, 26/II/1986, Lima and Gallindo 114, IPA 48526. s.l., XI/1936, Vasconcelos Sobrinho s.n., UFP 153.

Ocimum minimum L. *

Vernacular: Manjericão-miúda.
Native to Africa (Corrêa, 1926). Cultivated as medicinal in throughout Brazil.

Material examined: PERNAMBUCO: Recife, Arruda, 21/IV/1993, Albuquerque 16, UFP 8755.

\section{Ocimum tenuiflorum L. *}

Vernacular: Tulase.

Native to Asia, cultivated in Brazil (Albuquerque and Andrade, 1998a). Used as medicinal in the Old World (Nuñez and Castro, 1992).

Material examinado: PERNAMBUCO: Goiana, UEP de Itapirema, 12/II/1990, Lima 532, IPA 52495. Idem, 4/XI/1994, Cárceres 7, UFP 10711.

\section{Ocimum transamazonicum C. Pereira}

Brazilian species of the genus Ocimum. It is found inhabiting areas of savanna in the states of Maranhão, Pará, Pernambuco (forest zone) and Tocantins (Albuquerque and Andrade, 1998a; Albuquerque, 1998).

Material examined: PERNAMBUCO: Recife, Bongi, Arredores do IPA, 28/VI/1974, Andrade-Lima 74-7766, IPA 30189.

\section{Eriope Humb. and Bonpl. ex Benth. Eriope crassipes Benth.}

As with the entire genus, this species is widely distributed, showing great variation in morphology according to geographic environment (Harley, 1976). This species has been recorded from Pará, Mato grosso, Goiás, Brasília, Minas Gerais, Rio de Janeiro, São Paulo and Paraná, as well as some areas of Bahia (Harley, 1976). In Pernambuco occurred in caatinga zone.

Material examined: PERNAMBUCO: ArcoVerde, margem da estrada Ibimirim a Petrolândia, 13/XII/1954, Andrade-Lima 54, IPA 7562.

\section{Eriope cf. macrostachya Mart. ex Benth}

This species occur in mountain areas of southern Bahia, extending from Paraná to Paraguay (Harley, 1995). Occur in caatinga zone of Pernambuco.

Material examined: PERNAMBUCO: Buíque, Serra do Catimbau, 19/VIII/1994, Rodal 305, PEUFR 17387. 


\author{
Hypenia (Mart. ex Benth.) Harley \\ Hypenia salzmannii (Benth.) Harley \\ Vernacular: Alfazema-brava, barrigudinha.
}

It is a very frequent plant in the Caatinga, especially in mountain areas of northeastern Brazil, and is also recorded from Roraima (Harley, 1995). In Pernambuco, it was found from the forest zone to the caatinga zone.

Material examined: PERNAMBUCO: Afrânio, 1984, Pereira s.n., IPA 49887. Buíque, 25/V/1970, Xavier Filho s.n., UFP 2382. Idem, 21/X/1996, Baracho and Siqueira 245, UFP 22269. Idem, 18/VIII/1994, Rodal 266, PEUFR 17322. Gravatá, no campo, 18/IV/1983, Chiappeta s.n., IPA 31982. Idem, 22/X/1960, Tavares 539, UFP 514. Idem, VII/1926, Pickel s.n., IPA 4816. Ibimirim, 2/VI/1982, Ataide et al. s.n., IPA 28774. Ipubí, 10/VI/1992, Pereira et al. s.n., IPA 55017. Parnamirim, 11/VI/1984, Araújo s.n., IPA 42669. Idem, 11/VI/1984, Araújo s.n., PEUFR 7499. Petrolina, CODEVASF, 21/XII/1982, Fotius s.n., IPA 31546. Recife-Petrolina, 1979, Andrade-Lima et al. s.n., IPA 46460. Tacaratu, 6/X/1983, Gallindo et al. 26, IPA 43404. Tapera, Ipojuca, 15/X/1960, Miranda s.n., PEUFR 74. Trindade, 26/IV/1983, Gallindo et al. s.n., IPA 42572. Idem, $33 \mathrm{Km}$ após Ouricuri, 26/IV/1983, Gallindo et al. s.n., PEUFR 7375.

\section{Hyptis Jacq.}

\section{Hyptis atrorubens Poit.}

Vernacular: Hortelã-brava, hortelã-de-boi.

It is found on sandy fields near coastal zones, from Mexico and the Antilles to Brazil (north and northeastern) (Braga, s.d.). In Pernambuco occur from the forest zone to the caatinga zone.

Material examined: PERNAMBUCO: Caruaru, 10/IX/1971, Andrade-Lima 71-6484, IPA 25368. Maraial, Engenho Curtume, 13/X/1996, Siqueira Filho et al. 181, UFP 15751. Idem, Engenho Curtume, 30/VII/1997, Baracho 671/624 et al., UFP 22628. Palmares, 22/XI/1933, Pickel s.n., IPA 4807. Recife, Beberibe, IX/1936, Vasconcelos Sobrinho s.n., IPA 218. Idem, 3/VII/1958, Andrade-Lima 58-5950, IPA 21239. Idem, IX/1936, Vasconcelos Sobrinho s.n., PEUFR 154. São Bento do Una, VII/1926, Pickel s.n., IPA 4805. Idem, 7/IX/1931, Pickel s.n., IPA 4806. São Lourenço da Mata, 26/VII/1980, Andrade-Lima and Bocage 8, IPA 26626.
Hyptis brevipes Poit.

Vernacular: alfazema-braba, alfazema-brava

In Brazil it is distributed throughout the Northeast and is apparently restricted to the coast in the south (Harley, 1985). It was found in forest zone of Pernambuco

Material examined: PERNAMBUCO: Correntes, 4/XII/1985, Lima 41, IPA 48423.

\section{Hyptis calida Mart. ex Benth.}

In Brazil, it is distributed in the northeast. The collection place in Pernambuco was not specified.

Material examined: PERNAMBUCO: s.l., s.d., Pickersgill et al. s.n., IPA 20542.

\section{Hyptis fruticosa Salzm. ex Benth.}

In Brazil it is distributed in the northeast. In Pernambuco it was found from the forest zone to the caatinga zone.

Material examined: PERNAMBUCO: Buíque, Serra do Catimbau, 27/IV/1995, Albuquerque 63, UFP 11045. Idem, 27/IV/1995, Albuquerque 64, UFP 11029. Idem, 27/IV/1995, Albuquerque 68, UFP 11028. Idem, 27/IV/1995, Albuquerque 66, UFP 11024. Idem, 27/IV/1995, Albuquerque 65, UFP 11023. Idem, 27/IV/1995, Albuquerque 67, UFP 11030. Idem, Estrada Buíque-Catimbau. Sítio Bom Jesus, 21/X/1996, Baracho and Siqueira-Filho 272 et al., UFP 22002. Idem, Estrada Buíque-Catimbau, V/1995, Andrade et al. 36, PEUFR 18627. Idem, Serra do Catimbau, 16/IX/1994, Sales 367, PEUFR 17426. Idem, 17/X/1994, Sales 409, PEUFR 17426. Gravatá, 6/II/1966, Andrade-Lima 66-4460, IPA 13891. Recife, 2/II/1981, Ehrendorrfer 318, IPA 44597.

\section{Hyptis aff. lanceolata Poir.}

In Brazil it is distributed in the northeast. In Pernambuco occurs from the forest zone to the caatinga zone.

Material examined: PERNAMBUCO: Caruaru, Murici, Brejo dos Cavalos, Parque Ecológico Municipal, Costa e Silva 202, PEUFR 18362. Tapera, Ipojuca, 2/X/1931, Pickel s.n., IPA 4809. Idem, 22/IX/1924, Pickel s.n., IPA 7006. Idem, 30/XI/1927, Pickel s.n., IPA 7000. 


\section{Hyptis lantanifolia Poit.}

This species occur in north, southeast and northeastern Brazil. It occurs in coastal and forest zone of Pernambuco.

Material examined: PERNAMBUCO: Goiana, 30/V/1935, Pickel s.n., IPA 4811. Prazeres, Jaboatão, 9/VI/1933, Pickel s.n., IPA 4810.

\section{Hyptis lappacea Benth.}

According to available literature, this species occurs in the southern Brazil. In Pernambuco occurs in the forest zone.

Material examined: PERNAMBUCO: Recife, Curado, 17/VIII/1958, Andrade-Lima 58-5753, IPA 20994. Saltinho, Rio Formoso, 2/IX/1954, Falcão et al. 887, IPA 7872.

\section{Hyptis leucocephala Mart. ex Benth.}

In Brazil it is distributed in the northeast. In Pernambuco occurs in the caatinga zone.

Material examined: PERNAMBUCO: Afrânio, Margem da estrada, $2 \mathrm{Km}$ em direção ao Piauí, 22/IV/1971, Heringer et al. 285, IPA 19155. Petrolina, $4 \mathrm{Km}$ sul do CPATSA, 25/V/1983, Fotius 3485, IPA 31448 .

\section{Hyptis cf. macrostachys Benth.}

In Brazil it is distributed in the northeast. In Pernambuco occurs in the caatinga zone.

Material examined: PERNAMBUCO: Buíque, Chapada São José, 21/VI/1975, Andrade-Lima 75-8110, IPA 42963. Triunfo, 26/II/1986, Lima and Gallindo 71, IPA 48592.

\section{Hyptis martiusii Benth.}

This species occur in southeastern and northeastern Brazil. In Pernambuco occurs from the forest zone to the caatinga zone.

Material examined: PERNAMBUCO: Araripina, 25/VI/1952, Lima and Magalhães 52, IPA 5320. Idem, 27/IV/1983, Barreto et al. s.n., IPA 42597. Idem, 27/IV/1983, Barreto et al. s.n., PEUFR 7244. Buíque, 21/VI/1975, Andrade-Lima 75-8080, IPA 43000. Idem, 13/IX/1997, Pereira et al. 1139, IPA 56148. Idem, 19/VI/1994, Miranda et al. 1767, PEUFR 16800. Idem,
Estrada Buíque-Catimbau, Vegetação arbustiva densa, 16/VIII/1995, Andrade 137 and Figueredo, PEUFR 22701. Idem, Fazenda Laranjeiras, 20/V/1995, Laurêncio et al. 61, PEUFR 18633. Idem, Serra do Catimbau, Vegetação perenifolia arbustiva, 19/VIII/1994, Rodal 296, PEUFR 17320. Idem, Trilha das Torres, 19/X/1994, Rodal 416, PEUFR 17529. Idem, 16/IX/1994, Sales 362, PEUFR 17321. Idem, 18/X/1994, Marcon 9, PEUFR 17526. Idem, Fazenda Laranjeiras, 19/V/1995, Andrade et al. 46, PEUFR 18632. Idem, Estrada Buíque-Catimbau, 17/VIII/1995, Andrade 158 et al., PEUFR 23463. Idem, Serra do Catimbau, 22/IX/1995, Figueredo 204 et al., PEUFR 23275. Caruaru, 30/XI/1927, Pickel s.n., IPA 6973. Exu, 23/V/1980, Coradin et al. 2508, IPA 26765. Idem, 10/VI/1992, Pereira et al. 712, IPA 54977. Idem, 16/VI/1970, Xavier Filho s.n., UFP 2659. Garanhuns, 18/X/1949, Andrade-Lima 49-356, IPA 1372. ArcoVerde, 4/IX/1951, Lima 51-930, IPA 1372. Moxotó, 16/VII/1978, Gonçalves 26, IPA 30328. Serrita, 1/XII/1965, Vasconcelos Sobrinho 163, PEUFR 9870. Triunfo, 18/VI/1992, Ferraz 170, IPA 52923. Idem, 16/VI/1992, Ferraz 170, PEUFR 13451. Tupanatinga, 11/X/1977, Fonseca 10, IPA 51323.

\section{Hyptis paludosa St. Hil. ex Benth.}

From southeast to northeastern Brazil. It occurs in forest zone of Pernambuco.

Material examined: PERNAMBUCO: Prazeres, Jaboatão, 16/I/1935, Pickel 3759, IPA 7009.

\section{Hyptis pectinata (L.) Poit.}

Vernacular: Alfazema-brava, alfazema-de-caboclo.

It is a widely distributed plant, considered a weed. Its distribution ranges from the coast of Paraná northward throughout the tropical region of South and Central America, the Caribbean, to the United States of America. It was introduced into the tropics from Africa and Asia (Harley, 1985). In Pernambuco it occurs from the coastal zone to the caatinga zone.

Material examined: PERNAMBUCO: Belém de São Francisco, Ilha do Meio, 18/VII/1967, Tenório 67-311, IPA 14764. Buíque, 19/VIII/1994, Rodal 284, PEUFR 17468. Caruaru, Brejo dos Cavalos, 25/IV/1995, Albuquerque 46, UFP 11037. Idem, Brejo dos Cavalos, Mata arbórea densa, 22/XI/1995, Lucena 74 et al., PEUFR 25491. Idem, 3/XI/1995, Tschá 322 et al., PEUFR 25492. Correntes, Fazenda do Ministério da Agricultura, 3/XII/1985, Lima 12, IPA 48406. Exu, Serra do Araripe, 5/VIII/1986, Lima 292, IPA 49099. Idem, beira de estrada, 6/VIII/1986, Lima 299, IPA 
49104. Ferreiro, Usina Olho D’água, 4/X/1958, Andrade-Lima 58-5951, IPA 21238. Maraial, Paraíso, 5/VII/1965, Texeira s.n., PEUFR 9869. Idem, Engenho Curtume, 13/X/1996, Siqueira Filho 140 et al., UFP 15749. Paudalho, Granja Iranal, 7/IX/1986, Coelho 6, IPA 49059. Pesqueira, Serra do Ororobá, 22/III/1996, Albuquerque s.n., UFP 15593. Idem, Fazenda São Francisco, s.d., Correia 329, UFP 15676. Idem, 24/VII/1995, Correia 329, UFP 17903. Petrolina, CODEVASF, 15/VI/1983, Fotius 3492, IPA 31392. Idem, CODEVASF, 30/XII/1982, Fotius 3285, IPA 31472. Recife, s.d., Ramos s.n., UFP 1644. Idem, s.d., Ramos s.n., UFP 1645. Idem, Cidade Universitária, 15/X/1986, Barbosa s.n., UFP 4556. Idem, Curado, 3/VIII/1958, Andrade-Lima 58-5951, IPA 21240. Rio Formoso, Tamandaré, 26/VII/1954, Falcão et al. 784, IPA 7871. Idem, Tamandaré, 28/VII/1954, Falcão et al. 853, IPA 7873. São Lourenço da Mata Tapacurá, 1988, Alunos $2^{\circ}$. Semestre s.n., PEUFR 11998. Tapera, Ipojuca, 1928, Pickel s.n., IPA 7004. Taquaritinga do Norte, Sítio do Cafundó, 7/VI/1995, Albuquerque 79, UFP 11026. Idem, 7/VI/1995, Albuquerque 78, UFP 11027. Idem, 7/VI/1995, Albuquerque 80, UFP 11025.

\section{Hyptis platanifolia Mart. ex Benth.}

In Brazil it is distributed through the northeast. In Pernambuco occurs in the caatinga zone.

Material examined: PERNAMBUCO: Araripina, Chapada do Araripe, XII/1992, Carvalho Neto s.n., IPA 55889. Exu, Fazenda Dourado, Chapada do Araripe, 10/VI/1992, Pereira 722, IPA 55008.

\section{Hyptis pulegioides Pohl.}

From southeastern to northeastern Brazil. It was found in the forest zone in Pernambuco.

Material examined: PERNAMBUCO: Tapera, Campo Alegre, Ipojuca, 31/VIII/1936, Pickel s.n., IPA 4814. Idem, IX/1929, Pickel s.n., IPA 7014.

\section{Hyptis recurvata Poit.}

It is found from Amazonas to Rio de Janeiro and Minas Gerais (Corrêa, 1975). In forest zone of Pernambuco.

Material examined: PERNAMBUCO: Prazeres, Jaboatão, 16/I/1935, Pickel s.n., IPA 4815.

\section{Hyptis suaveolens (L.) Poit.}

Vernacular: bamburral, alfazema-de-caboclo, alfazema-brava, alfavaca-brava.
It inhabits open areas of the Caatinga, from the mountain foods to the coast. It is also found in America, Tropical Asia (Corrêa, 1974; Braga, s.d.), Mexico and the Caribbean south to Brazil and Bolivia (Harley, 1995). In Pernambuco occur from the coastal zone to the caatinga zone.

Material examined: PERNAMBUCO: Afrânio, 23/IV/1971, Heringer et al. s.n., IPA 19167. Bucú, 2/IV/1934, Luetzelburg s.n., IPA 21723. Exu, 6/VIII/1986, Lima 306, IPA 49199. Fernando de Noronha, 18/X/1955, Andrade-Lima 55-2190, IPA 8237. Flores, 25/V/1971, Heringer et al. s.n., IPA 19809. Idem, 25/IV/1983, Gallindo et al. s.n., IPA 42568. Idem, 25/V/1971, Heringer et al. s.n., PEUFR 5468. Idem, Vilarejo de Barragem, 25/IV/1983, Gallindo et al. s.n., PEUFR 7266. Gravatá, VII/1926, Pickel s.n., IPA 4817. Novo Exu, 2/VIII/1934, Luetzelburg s.n., IPA 22580. Parnamirim, 18/V/1984, Araújo 53, IPA 42033. Idem, 20/VI/1984, Araújo 167, IPA 42707. Idem, Km 27, 18/V/1984, Araújo 53, UFP 6353. Idem, Km 27, 20/VI/1984, Araújo s.n., IPA 7470. Paulista, Mata do Caetés, XI/1992, Gomes s.n., PEUFR 21812. Serra Talhada, 15/IV/1986, Ataide 635 , IPA 48781.

\section{Hyptis umbrosa Salzm. ex Benth.}

Vernacular: Hortelã-do-mato, bamburral.

According to Harley (1985) it is a ruderal from southern Brazil, from Vale do Ribeira (Paraná) to Vale do Itajaí (Santa Catarina), and extending into Minas Gerais and Goiás (Harley, 1995). In Pernambuco occur from the forest zone to the caatinga zone.

Material examined: PERNAMBUCO: Bezerros, Parque Ecológico de Serra Negra, Vegetação arbustivaarbórea, 5/X/1995, Silva 64 et al., PEUFR 21382. Bonito, Reserva Ecológica Municipal da Prefeitura de Bonito, 15/II/1995, Villarouco 2 et al., PEUFR 1866. Idem, 15/III/1995, Tschá et al. 34, PEUFR 19157. Brejo da Madre de Deus 15/IX/1973, Andrade-Lima 73-7475, IPA 29590. Idem, 15/VI/1973, Andrade-Lima 73-7401, IPA 29756. Idem, Fazenda Buriti, Solo pedregoso, 24/V/1995, Villarouco 100 et al., PEUFR 18954. Maraial, Engenho Curtume, 13/X/1996, Siqueira Filho 173 et al., UFP 15748.

\section{Marsypianthes Mart. ex Benth. Marsypianthes chamaedrys (Vahl) Kuntze Vernacular: Meladinha.}

It is found in the Antilles, Mexico, Central and South America (Braga, s.d.). It is a plant of the 
equatorial zone of Brazil. In Pernambuco it occurs from the coastal zone to the caatinga zone.

Material examined: PERNAMBUCO: Belém de São Francisco, Ilha do Meio, 20/IV/1967, Tenório 67-326, IPA 14779. Buíque, Chapada São José, 21/VI/1975, Andrade-Lima 75-8089, IPA 46225. Campo Alegre, Ipojuca, 3/III/1925, Pickel s.n., IPA 7002. Caruaru, 23/X/1927, Pickel s.n., IPA 4822. Gravatá, VII/1926, Pickel s.n., IPA 4821. Ibimirim, 2/VI/1982, Ataide et al. 38, IPA 30034. Igarassu, 21/VIII/1954, Falcão et al. 755, IPA 7875. Olinda, 1916, Pickel s.n., IPA 7133. Parnamirim, Km 5, 27/VII/1984, Araújo 22, IPA 42037. Idem, Solo litólico, Substrato micaxisto, 27/IV/1984, Araújo s.n., PEUFR 7511. Paudalho, Granja Iranal, 7/IX/1986, Coelho 5, IPA 49060. Petrolina, CODEVASF, Área irrigada em Alqueiro, 14/XII/1982, Fotius 3217, IPA 31464. Idem, Área do CPATSA, 24/VII/1984, Pinto 143/84, IPA 41794. Recife, Bongi, III/1936, Vasconcelos Sobrinho s.n., IPA 92. Idem, Curado, Campo de cultura, 4/X/1958, Andrade-Lima 58-5763, IPA 21004. Idem, Dois Irmãos, COMPESA, 19/X/1985, Gallindo 127, IPA 48477. Idem, Bongi, III/1936, Vasconcelos Sobrinho s.n., UFP 155. Idem, 5/VIII/1961, Tavares 741, UFP 629. Idem, Cidade Universitária, 19/III/1985, Silva s.n., UFP 4304. Serra Talhada, Proximidades do Hotel Planalto, IV/1971, Heringer et al. s.n., IPA 19520. Idem, Serra da Carnaubeira, 22/V/1971, Heringer et al. s.n., IPA 19731. Idem, Serra da Carnaubeira, 22/V/1971, Heringer et al. s.n., PEUFR 4212. Idem, Hotel Planalto, 22/V/1971, Heringer et al. s.n., PEUFR 6185. Tapera, Ipojuca, IV/1928, Pickel s.n., IPA 4823. Idem, 26/VI/1934, Pickel s.n., IPA 7015. Triunfo, Divisa Triunfo-Princesa, 25/II/1986, Lima and Gallindo 17, IPA 48397.

\section{Raphiodon Schauer Raphiodon echinus (Nees and Mart.) Schauer}

Distributed along eastern Brazil. Widespread in northeastern Brazil (Harley, 1995). In Pernambuco occurs from the forest zone to the caatinga zone.

Material examined: PERNAMBUCO: Araripina, XII/1992, Carvalho Neto s.n., IPA 55877. Buíque, 12/IX/1997, Pereira et al. 1081, IPA 56146. Idem, 19/IX/1969, Tenório 69-1141, IPA 20391. Idem, 18/X/1994, Villarouco 9, PEUFR 18301. Idem, 17/VIII/1995, Figueredo 139 et al., PEUFR 22702. Idem, 23/II/1996, Andrade 318 et al., PEUFR 23464. Idem, Sopé de Serra, Vegetação arbustiva esparsa, 12/I/1996, Campelo 28 et al., PEUFR 23508. Idem, 18/VIII/1994, Rodal 271, PEUFR 18749. Carnaubeira (Floresta)-Conceição das Crioulas 18/V/1971, Heringer et al. s.n., PEUFR 4332. Condado, 6/VIII/1992, Miranda et al s.n., PEUFR 12379.
Ibimirim, 2/VI/1982, Ataide et al. 10, IPA 28752. També, s.d., Vasconcelos Sobrinho 9-937, IPA 618. Floresta, 10/V/1971, Heringer et al. s.n., IPA 19702. Ouricuri-Parnamirim, 7/V/1971, Heringer s.n., IPA 19511. Petrolina, 14/VI/1983, Fotius 3491, IPA 31354. Recife, 2/I/1981, Ehrendorrfer 3181, IPA 44603. Idem, 2/I/1981, Ehrendorrfer 318, IPA 44604. Serra Talhada, 10/V/1971, Heringer et al. s.n., IPA 19562. Serra Talhada-Salgueiro, 10/V/1971, Heringer s.n., PEUFR 6143. Tapera, Ipojuca, 15/VI/1935, Pickel 3870, IPA 7010.

\section{Aeollanthus Mart. ex Spreng. Aeollanthus suaveolens Mart. ex Spreng. * Vernacular: Macassá.}

Of African origin. Cultivated as medicinal throughout Brazil.

Material examined: PERNAMBUCO: Recife, II/1985, Pires 6, UFP 4270. Tapera, Ipojuca, 2/XI/1932, Pickel s.n., IPA 4825.

\section{Plectranthus L'Hér.}

Plectranthus amboinicus (Lour.) Spreng *

Vernacular: Hortelã-graúda.

Native to South Oriental Asia. It is found from the Antilles to Southern Brazil (Carriconde et al., 1996). Cultivated as medicinal.

Material examined: PERNAMBUCO: Caruaru, SESC, 9/V/1997, Farias s.n., UFP 17970. Goiana, 1990, Lima 522, IPA 52502. Idem, 31/X/1991, Lima 539, IPA 52553. Recife, Ibura, 19/VIII/1991, Krush s.n., PEUFR 11536. Idem, Vasco da Gama, 2/VII/1985, Silva s.n., UFP 4429. Tapera, Ipojuca, 5/II/1991, Pickel s.n., IPA 4827. Idem, V/1927, Pickel s.n., IPA 4826. Idem, s.d., Pickel s.n., IPA 4804.

\section{Plectranthus barbatus Andr. *}

Vernacular: Falso-boldo, boldo-do-reino, malvasanta, tapete-de-oxalá, sete-dores.

Perennial shrub, probably of African origin (Santos et al., 1988). Cultivated as medicinal.

Material examined: PERNAMBUCO: Recife, Curado, Km 12, PE 232, 13/I/1997, Farias s.n., UFP 16270.

\section{Solenostemon Thonn. \\ Solenostemon scutellarioides (L.) Codd *}

Cultivated as ornamental throughout northeastern Brazil. 
Material examined: PERNAMBUCO: Nova Cruz, Jardim Residencial, s.d., Gomes s.n., PEUFR 11864. Recife, Dois Irmãos, 7/VIII/1993, Gomes and Eduardo s.n., PEUFR 14550. Idem, Campo Grande, 10/X/1960, Miranda s.n., PEUFR 75. Tapera, Ipojuca, 1/VIII/1924, Pickel s.n., IPA 4828.

\section{Tetradenia Benth.}

Tetradenia riparia (Hochst.) Codd *

Vernacular: Mirra.

Species of African origin. Cultivated as ornamental and medicinal.

Material examined: PERNAMBUCO: Recife, Vasco da Gama, 5/VII/1985, Silva s.n., UFP 4425.

\section{Subfamily Pogostemonoideae Pogostemon Desf.}

Pogostemon patchouly Pellet *

Vernacular: Patcholi, patchouli.

Native to India and Burma (Corrêa apud Di Stasi et al., 1989). Cultivated in Pernambuco.

Material examined: PERNAMBUCO: Goiana, UEP de Itapirema, 22/VI/1992, Azevedo 4, IPA 52794.

\section{ACKNOWLEDGMENTS}

The authors like to thanks to Dr. William Sanders, Departamento de Botânica, Universidade Federal de Pernambuco, for invaluable help with the revision of the english text.

\section{RESUMO}

O levantamento da família Lamiaceae no estado de Pernambuco revelou a existência de 20 gêneros aos quais estão subordinadas 46 espécies. Os gêneros com maior número de exsicatas foram Hyptis e Ocimum, outros tiveram poucos materiais representativos, existindo aqueles coletados uma única vez. Com relação a distribuição fitogeográfica no estado, $9,1 \%$ dos registros de espécies são da Zona do Litoral, 54,5\% da Zona da Mata e 36,3\% da Zona da Caatinga.

\section{REFERENCES}

Albuquerque, U. P. and Andrade, L. H. C. (1998a), El género Ocimum L. (Lamiaceae) en el Nordeste del Brasil. Anales del Jardin Botánico de Madrid, 56 : (1), 43-64.

Albuquerque, U. P. and Andrade, L. H. C. (1998b.), Etnobotánica del género Ocimum L. en las comunidades afrobrasileñas. Anales del Jardin Botánico de Madrid, 56 : (1), 107-118.

Albuquerque, U. P. (1998), Morphological and taxonomical notes on Ocimum transamazonicum C. Pereira. Naturalia, 23, 55-60.

Albuquerque, U. P. (1999), Three new varieties in Ocimum L. (Lamiaceae). Brazilian Archives of Biology and Technology, 42 : (1), 01-08.

Andrade-Lima, D. (1960), Estudos fitogeográficos de Pernambuco. 2.ed. Arquivo do Instituto de Pesquisas Agronômicas, 5, 305-341.

Andrade-Lima, D. (1966), Contribuição ao estudo do paralelismo da flora amazônica-nordestina. Boletim Técnico do IPA, 19, 1-30.

Braga, R. [S.d.], Plantas do nordeste - Especialmente do Ceará. 4. ed. Rio Grande do Norte. Editora Universitária do UFRN.

Carriconde, C.; Mores, D.; Fritschen, M. von and Júnior, E. L. C. (1996), Plantas medicinais and alimentícias. Centro Nordestino de Medicina Popular: UFRPE, Olinda. v. 1.

Castro, L. O. and Chemale, V. M. (1995), Plantas medicinais, condimentares e aromáticas: descrição $e$ cultivo. Agropecuária, Guaíba.

Corrêa, M. P. (1926), Dicionário das plantas úteis do Brasil e das exóticas cultivadas. Imprensa Nacional, Rio de Janeiro. v. 1.

Corrêa, M. P. (1969), Dicionário das plantas úteis do Brasil e das exóticas cultivadas. Imprensa Nacional, Rio de Janeiro. v. 4.

Corrêa, M. P. (1974), Dicionário das plantas úteis do Brasil e das exóticas cultivadas. Imprensa Nacional, Rio de Janeiro. v. 5.

Corrêa, M. P. (1975), Dicionário das plantas úteis do Brasil e das exóticas cultivadas. Imprensa Nacional, Rio de Janeiro. v. 6.

Di Stasi, L. C.; Santos, E. M. G.; Santos, C. M. and Hiruma, C. A. (1989), Plantas medicinais na Amazônia. Editora Universidade Estadual Paulista, São Paulo.

Epling, C. and Toledo, J. F. (1943), Labiadas. In- Flora Brasílica. Secretaria da Agricultura, Indústria e Comércio de São Paulo. v. 48, fasc. 7.

Harley, R. M. (1976), Hooker's icones plantarum - A review of Eriope and Eriopidion (Labiatae). Vol. 38. Parte III. Royal Botanic Garden, Kew, London.

Harley, R. M. (1985), Flora Ilustrada Catarinense: $1^{a}$ parte. Raulino Reitz, Santa Catarina. 
Harley, R. M. (1988), Evolution and distribution of Eriope (Labiatae) and its relatives in Brasil. In- Vanzolini, P.E. and Heyer, W.R. (eds). Proceedings of a workshop on Neotropical Distribution Patterns. Academia Brasileira de Ciências. Rio de Janeiro. pp. 71-80.

Harley, R. M. (1995), Labiatae - Flora of the Pico das Almas, Chapada Diamantina, Bahia, Brazil. B. L. Stannard (ed.) Royal Botanic Garden, Kew, London

Harley, R. M. (1996), The Labiatae of Bahia: A prelimirary check-list. Sitientibus, Feira de Santana, 15, 11-21.

Heywood, V. H. (1978), Flowering plants of the world. Oxford University Press, London.

Hedge, I. C. (1992), A global survey of the biogegraphy of the Labiatae. In- Harley, R. M. and Reynolds, T. (eds). Advances in Labiatae Science. pp. 7-17.

Holmgren, P. K.; Holmgrem, N. H. and Barnet, L. C. (1990). Index Herbariorum, Part. 1: The Herbaria of the World. 8.ed. Botanical Garden, New York.

Lainetti, R. and Brito, N. R. S. (1980), A saúde pelas plantas e ervas do mundo inteiro. Rio de Janeiro : Ediouro.
Morgan, R. (1997), Enciclopédia das ervas e plantas medicinais. 8. ed. São Paulo : Hemus Editora.

Nuñes, D. R. and Castro, C. O. (1992), The ethnobotany of old world Labiatae. In- Harley, R. M. and Reynolds, T. (eds). Advances in Labiatae Science. pp. 455-473.

Pereira, C. (1965), Flora do estado da Guanabara. Labiatae. Arq. Jard. Bot., 18, 87-108.

Pereira, C. and Pereira, E. (1973), Flora do Estado do Paraná. Labiatae. Arq. Jard. Bot., 19, 79-108.

Silva, G. C. (1985), Flora e vegetação das depressões inundáveis da região de Ouricuri-PE. Dissertação de Mestrado, Universidade Federal Rural de Penambuco, Recife.

Santos, C. A. M.; Torres, K. R. and Leonart, R. (1988), Plantas medicinais: Herbarium, flora et scientia. 2. ed. São Paulo : Editora Ícone.

Received: April 19, 2000; Revised: March 06, 2001; Accepted: September 24, 2001. 